\title{
The Higgs boson at the LHC: standard model Higgs properties and beyond standard model searches
}

\author{
Anne-Marie Magnan*广 \\ Imperial College London \\ E-mail: a.magnan@imperial.ac.uk
}

\begin{abstract}
After the discovery of a new particle in 2012 by the ATLAS and CMS collaborations, since then identified to be the standard model Higgs boson, a brief review is made of recent results using LHC data taken at a centre-of-mass energy of $13 \mathrm{TeV}$ in 2016. The SM Higgs boson properties continue to be studied with greater precision, and all production modes and decay channels are probed, including the production in association with top quark, whose $5 \sigma$ observation is made possible by the data accumulated so far. New searches for rare and exotic decays of the Higgs boson are presented, as well as searches for additional neutral and charged Higgs bosons. Recent di-Higgs boson production results are also briefly described.
\end{abstract}

An Alpine LHC Physics Summit (ALPS2018)

15-20 April, 2018

Obergurgl, Austria

\footnotetext{
*Speaker.

${ }^{\dagger}$ on behalf of the ATLAS and CMS collaborations
} 


\section{Introduction}

These proceedings are dedicated to reviewing the most recent results on the Higgs boson at the Large Hadron Collider, from the ATLAS and CMS collaborations, using the data recorded in 2016. The ATLAS and CMS detectors are described in detail in [1] and [2], respectively.

The luminosities recorded by ATLAS and CMS over the past years are shown in Figure 1. The run-I data-taking period refers to datasets taken in 2010 to 2012 at 7 and $8 \mathrm{TeV}$ of centre-of-mass energies, whereas run-II refers to data taken in 2015 and 2016 at $13 \mathrm{TeV}$ of centre-of-mass energy.
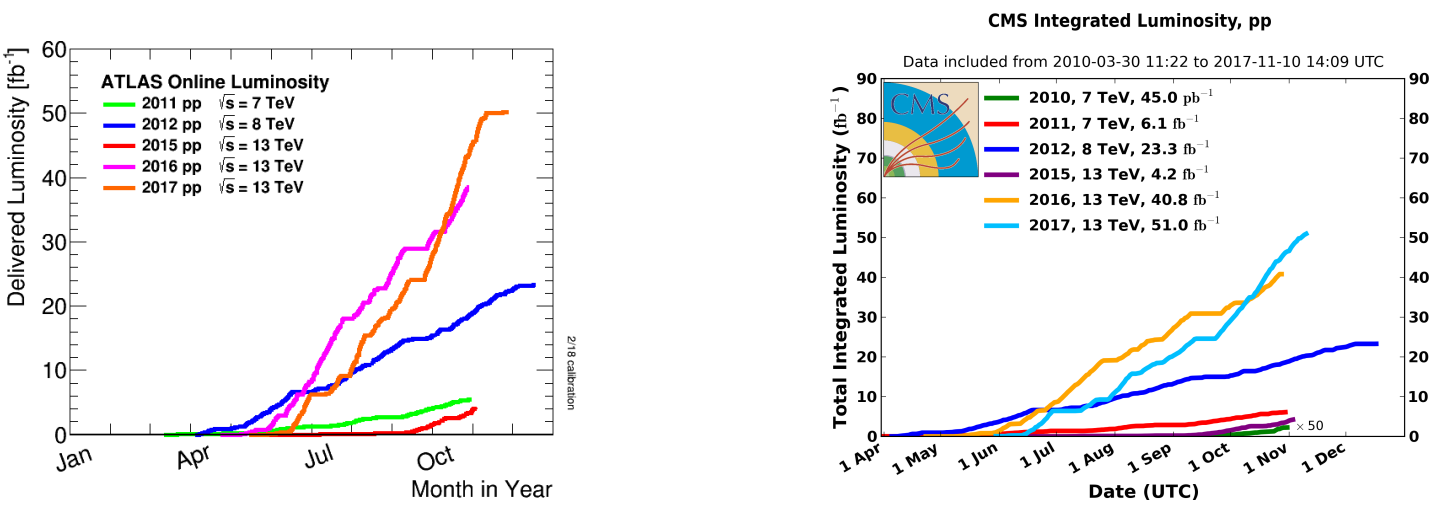

Figure 1: Integrated luminosities recorded in the ATLAS (left) and CMS (right) experiments. From [3] and [4] respectively.

The review is organised in several sections, and concerns both Standard Model (SM) Higgs boson properties and Beyond Standard Model (BSM) searches. In BSM searches, results from exotic decay channels as well as additional neutral and charged Higgs bosons are considered. The reader is referred to the references for more details about the way the analyses are performed and their complete sets of results.

\section{SM Higgs boson properties}

The Higgs boson was discovered in July 2012, using $\gamma \gamma$ and diboson (VV) final states, with data recorded at centre-of-mass energies of 7 and $8 \mathrm{TeV}[5,6]$. The new particle was further characterised by the measurement of its coupling to tau leptons, with the 5- $\sigma$ observation on the run-I dataset with the combination of ATLAS and CMS data [7], and by a single experiment in July 2017 with $7+8+13$ (2016) TeV datasets [8]. The evidence of the coupling to b quarks happened in the summer 2017 also with the combination of the 7+8+13 (2016) TeV datasets by both ATLAS and CMS separately $[9,10]$. The $3-\sigma$ evidence of the coupling to top quark was released by ATLAS in December 2017 with the $201613 \mathrm{TeV}$ dataset [11] followed by the 5- $\sigma$ observation by CMS the week before the ALPS 2018 conference with 7+8+13 (2016) TeV datasets [12].

The Higgs boson mass has been measured using the golden decay channels to $\mathrm{ZZ}$ to 4 leptons and $\gamma \gamma$ : these give the best resolutions and narrow mass distributions. The run-I legacy results for the mass measurements are in [13], and run-II updates have been released by ATLAS [14] and CMS [15], as summarised in Table 1. 


\begin{tabular}{l|l} 
LHC Run I & $125.09 \pm 0.21($ stat $) \pm 0.11($ sys $) \mathrm{GeV}$ \\
\hline ATLAS 2016 & $124.98 \pm 0.19($ stat $) \pm 0.21($ sys $) \mathrm{GeV}$ \\
CMS 2016 & $125.26 \pm 0.20($ stat $) \pm 0.08($ sys $) \mathrm{GeV}$ \\
\hline
\end{tabular}

Table 1: Summary of the Higgs boson mass measurements and their statistical (stat) and systematic (sys) accuracies, from $[13,14,15]$.

The spin and parity measurements were studied extensively using the same golden channels with run-I data, with the quantum numbers $\mathbf{J}^{P}=0^{+}$confirmed by data. The measurements are used to set severe constraints on anomalous couplings, see [16],[17].

In order to confirm further that the particle found is the SM Higgs boson, its couplings to the different particles are measured more and more precisely. The channels considered are shown in Figure 2 for ATLAS (left) and CMS (right), both in terms of production and decay modes. The red squares highlight the combinations that have been performed already with the run-II data.

\begin{tabular}{|l|c|c|c|c|}
\hline & ggF & VBF & VH & ttH \\
\hline $\mathbf{H} \rightarrow \mathbf{Z Z \rightarrow \text { 4I }}$ & $\bullet$ & $\bullet$ & $\bullet$ & $\bullet$ \\
\hline $\mathbf{H} \rightarrow \mathbf{Y Y}$ & $\bullet$ & $\bullet$ & $\bullet$ & $\bullet$ \\
\hline $\mathbf{H} \rightarrow \mathbf{W W}$ & $\bullet$ & $\bullet$ & & $\bullet$ \\
\hline $\mathbf{H} \rightarrow \mathbf{b b}$ & & $\bullet$ & $\bullet$ & $\bullet$ \\
\hline $\mathbf{H} \rightarrow \mathbf{T}$ & & & & $\bullet$ \\
\hline $\mathbf{H} \rightarrow \boldsymbol{\mu} \boldsymbol{\mu}$ & $\bullet$ & $\bullet$ & & \\
\hline $\mathbf{H} \rightarrow \mathbf{i n v}$ & & & $\bullet$ & \\
\hline
\end{tabular}

\begin{tabular}{|l|c|c|c|c||}
\hline & ggF & VBF & VH & ttH \\
\hline$H \rightarrow Z Z \rightarrow$ 4I & $\bullet$ & $\bullet$ & $\bullet$ & $\bullet$ \\
\hline$H \rightarrow$ YY & $\bullet$ & $\bullet$ & $\bullet$ & $\bullet$ \\
\hline$H \rightarrow W W$ & $\bullet$ & $\bullet$ & $\bullet$ & $\bullet$ \\
\hline$H \rightarrow$ bb & $\bullet$ & & $\bullet$ & $\bullet$ \\
\hline$H \rightarrow T \tau$ & $\bullet$ & $\bullet$ & & $\bullet$ \\
\hline$H \rightarrow \boldsymbol{\mu}$ & $\bullet$ & $\bullet$ & & \\
\hline$H \rightarrow$ inv & $\bullet$ & $\bullet$ & $\bullet$ & \\
\hline
\end{tabular}

Figure 2: Decay channels and production modes considered for the measurements of the Higgs boson couplings to SM particles. Left: ATLAS. Right: CMS. The red squares highlight the combinations that have been performed already with the 2016 data. Courtesy of Andrew Gilbert [18].

The run-I results from ATLAS and CMS data combined are described in details in [7], and shown in Figure 3, left. The full combination of 2016 data from CMS is shown in Figure 3, right, from [22]. Several new channels have been added compared to the run-I combination, namely $\mathrm{ZZ}$ decay for associated production modes, the search for a boosted $\mathrm{b} \overline{\mathrm{b}}$ pair targeting gluon-fusion production and decay to $\mathrm{b}$ quarks [19]. The best accuracy to date is obtained for $\mathrm{ggH}(11 \%), \mathrm{ttH}$ (26\%), and the full combination (9\%). ATLAS results using ZZ to $4 \ell$ and $\gamma \gamma$ decays are described in [20], and using WW decay in [21].

Differential measurements have also been done, to characterise further the kinematic properties of the Higgs boson in comparison with several theoretical calculations. The golden $\mathrm{H} \rightarrow \gamma \gamma$ and $\mathrm{H} \rightarrow 4 \ell$ decays are used by ATLAS [23] and the data are compared with Monte Carlo (MC) predictions for gluon fusion $(\mathrm{ggH})$, vector-boson fusion $(\mathrm{VBFH})$, and vector-boson associated (VH) production from Powheg-box v2, and for $\mathrm{ttH}$ and bbH production from Madgraph5_aMC@NLO. All predictions are normalised to the latest calculations (e.g. ggH N3LO). Statistical uncertainties are still dominant at the level of 20-30\%. A good agreement is obtained between both decay channels $\gamma \gamma$ and $4 \ell$, and with predictions.

The first evidence of ttH production was released by ATLAS in [11], from multileptons analy- 

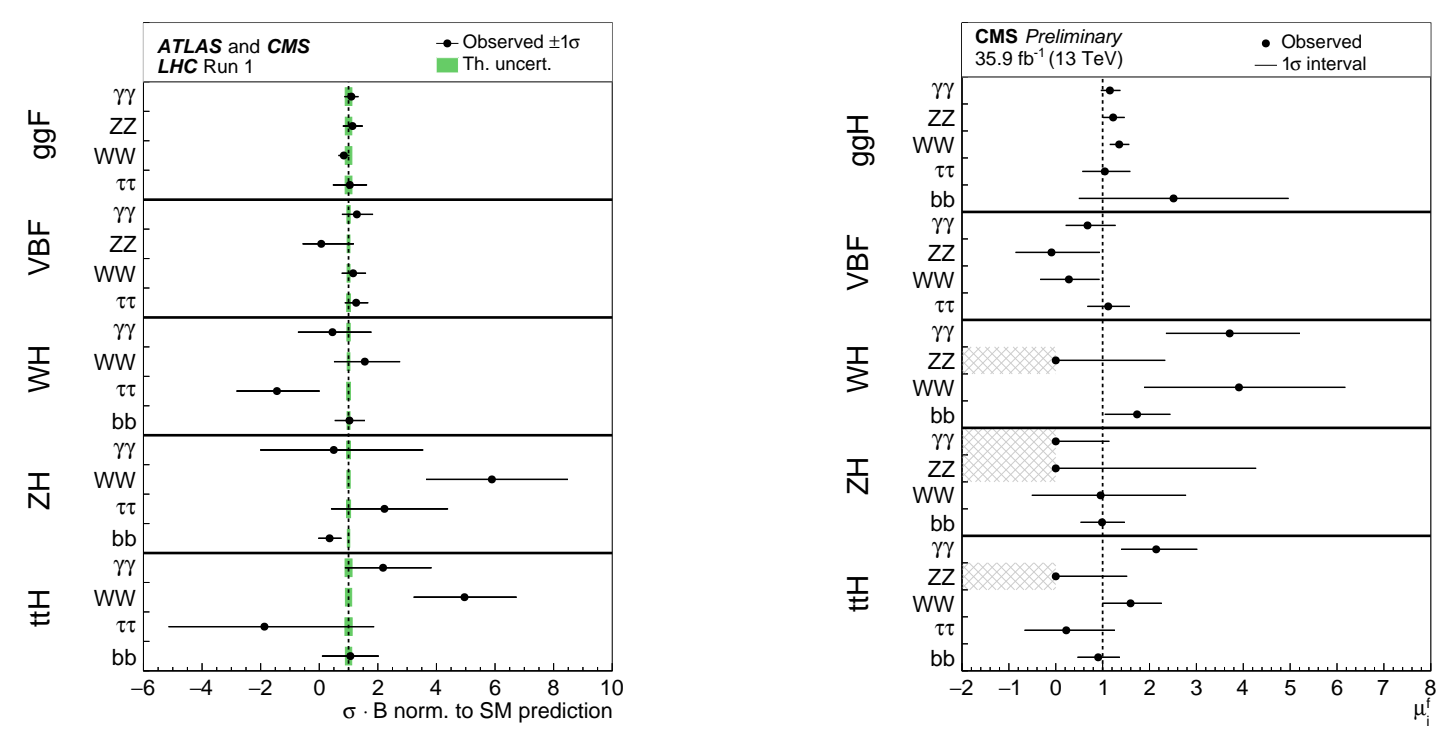

Figure 3: Observed signal strengths, relative to SM expectations, classified by decay channels and production modes. Left: run-I combined ATLAS and CMS results, from [7]. Right: CMS run-II combination, from [22].

ses, with a signal strength of $1.2 \pm 0.2$ (stat) \pm 0.2 (sys). The $201613 \mathrm{TeV}$ data were used targeting $\mathrm{WW}, \mathrm{ZZ}$, and $\tau \tau$ decay modes. In the CMS paper [12] showing the first observation of ttH production, 88 categories have been combined using the full statistics of 7, 8 and $13 \mathrm{TeV}$ data, and targeting WW, ZZ, $\tau \tau$, bb and $\gamma \gamma$ decays. The signals strength measured by CMS is:

$$
\mu_{t t H}=1.26_{-0.26}^{+0.31}=1.26_{-0.16}^{+0.16}(\text { stat })_{-0.15}^{+0.17}(\text { expt })_{-0.13}^{+0.14}(\text { bkg th })_{-0.07}^{+0.15}(\text { sig th })
$$

The observed and expected significances from both experiments are summarised in Table 2.

\begin{tabular}{|l|c|c|}
\hline Significance & ATLAS & CMS \\
\hline Observed & $4.2 \sigma$ & $5.2 \sigma$ \\
Expected & $3.8 \sigma$ & $4.2 \sigma$ \\
\hline
\end{tabular}

Table 2: Observed and expected significances for the measurement of $\mathrm{ttH}$ production in ATLAS and CMS. From [11, 12].

The combined CMS coupling measurements allow to put new constraints on the branching ratio of the Higgs boson to invisible decay products, set at BR(inv) $<0.22 @ 95 \% \mathrm{CL}$, and on various two-Higgs-doublet models (2HDM), see [22].

\section{Rare decays}

Having studied already in details the decay of the Higgs boson to b quarks, the next step is to look at the decay to c quarks. This channel, in addition to having a more than 10 times smaller branching ratio than the $b \bar{b}$ channel, is also extremely challenging for the experiments. Using the VH production, ATLAS gives an observed (expected) upper limit on the cross section 
times branching ratio of $\sigma<2.7\left(3.9_{-1.1}^{+2.1}\right) \mathrm{pb}$ [24]. The corresponding observed (expected) signal strength is $\mu<110\left(150_{-40}^{+80}\right)$.

Concerning the decay to two muons, the expected branching ratio is another two order of magnitudes smaller but the signal is extremely clean experimentally and benefiting from excellent performances of the detector in reconstructing the dimuon invariant mass. The upper limits are approaching the SM prediction with the ATLAS observed (expected) upper limit at 2.8 (2.9) times the SM prediction [25], and the CMS observed (expected) upper limit at 2.64 (1.89) times the SM prediction [26].

With a similar branching ratio than the Higgs boson to $\gamma \gamma$ decay, ATLAS and CMS have also studied the decay to a dilepton pair (from a $\mathrm{Z}$ boson) and a photon. Interpretations are made also for high mass spin-0 or spin-2 resonances. ATLAS sets an observed (expected) $95 \% \mathrm{CL}$ upper limit at 6.6 (5.2) times SM [27]. CMS sets an observed (expected) 95\%CL upper limit at 3.9 (2.0) times SM [28]. CMS gained in sensitivity by also considering offshell lepton pair production.

Finally decay channels involving mesons have been studied: $\mathrm{H} \rightarrow \phi \gamma, \rho \gamma$. These channels allow to test the coupling of the Higgs boson to light quarks [29], with diagrams as shown Figure 4, left. The upper limits on the branching ratios are shown in Figure 4, right. These recent ATLAS results complement the run-I $\mathrm{H} \rightarrow J / \Psi+\gamma$ searches presented in [30] and [31].

\begin{tabular}{c|c|c}
\hline Branching Fraction Limit (95\% CL) & Expected & Observed \\
\hline $\mathcal{B}(H \rightarrow \phi \gamma)\left[10^{-4}\right]$ & $4.2_{-1.2}^{+1.8}$ & 4.8 \\
$\mathcal{B}(Z \rightarrow \phi \gamma)\left[10^{-6}\right]$ & $1.3_{-0.4}^{+0.6}$ & 0.9 \\
$\mathcal{B}(H \rightarrow \rho \gamma)\left[10^{-4}\right]$ & $8.4_{-2.4}^{+4.1}$ & 8.8 \\
$\mathcal{B}(Z \rightarrow \rho \gamma)\left[10^{-6}\right]$ & $33_{-9}^{+13}$ & 25 \\
\hline
\end{tabular}

Figure 4: Left: representative diagrams for the decay of the Higgs boson to a photon and a meson. Right: observed and expected 95\%CL upper limits on the branching fractions of the Higgs and Z bosons to $\phi \gamma$ and $\rho \gamma$. From [29].

\section{Exotic decays}

Having studied already almost all possible expected SM decay channels, signs of new physics can also be looked for in exotic modes. With the run-I data, a $2.4 \sigma$ excess was observed by CMS in [32] for the lepton-flavour violating decay in the $\mu \tau$ final state. Unfortunately this is now ruled out by the 2016 data, see [33].

In many BSM models, the Higgs boson can decay to invisible particles. Complementing the indirect limits from visible decays, the direct search targets VH, ggH and VBFH production modes [34]. The 95\% CL upper limit on the branching fraction to invisible assuming SM Higgs boson production is shown in Figure 5, left. The VBFH channel is the most sensitive.

The Higgs boson could also decay to new particles like dark photons, through different portals (Hypercharge portal or Higgs portal), or light pseudoscalars. This is looked for in a final state with 4 leptons in [35], from either a $\mathrm{Z}$ boson and new vector $\mathrm{Z}_{D}$ (or pseudoscalar a) or two new $\mathrm{Z}_{D}$. The interpretation is given in several models, including $\mathrm{H} \rightarrow a a \rightarrow 4 \ell$. 

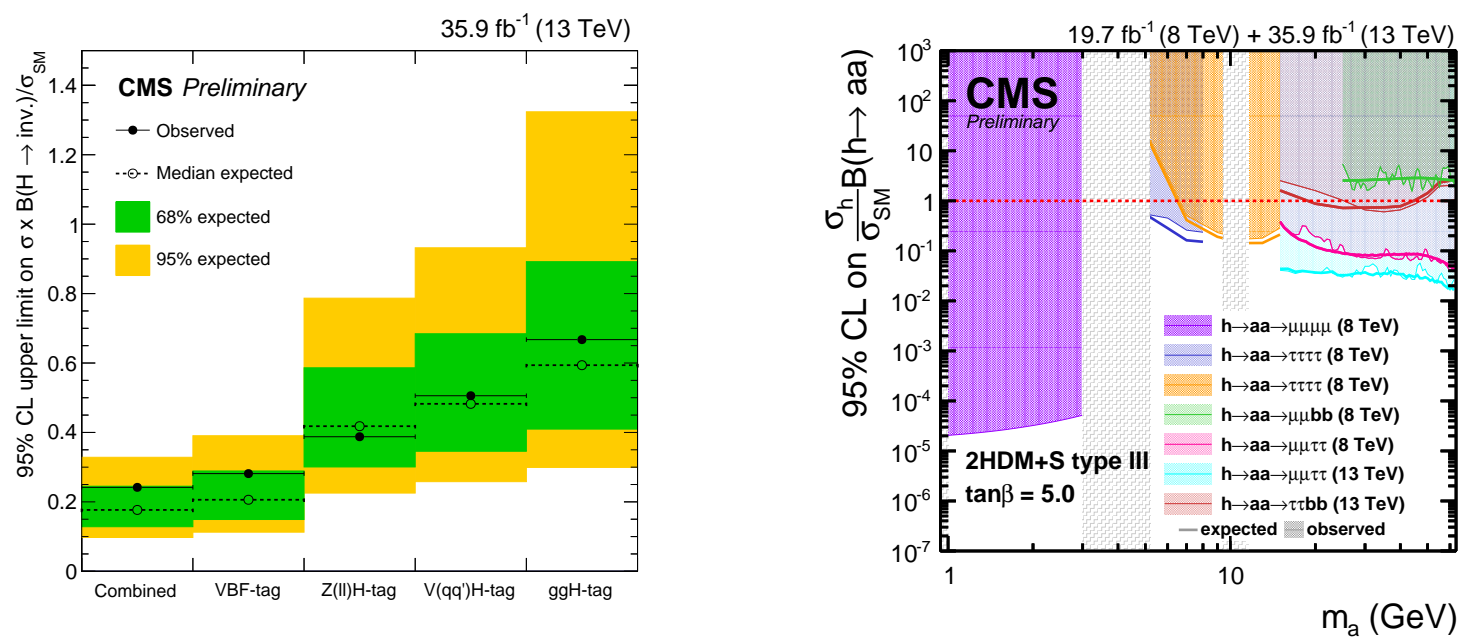

Figure 5: Left: 95\% CL upper limit on the branching fraction to invisible assuming SM Higgs boson production for different production modes and the combination, from [34]. Right: 95\% CL upper limit on the branching fraction to two a boson as a function of the a boson mass, in a type-III $2 \mathrm{HDM}+\mathrm{S}$ model with $\tan \beta=5$, from [38].

\section{Decays to lighter (pseudo)scalar particles}

In extended Higgs sector models like 2HDM with an additional singlet (2HDM+S), extra light scalars / pseudoscalars (a) are predicted. For a masses below half the mass of the SM Higgs boson, the decay channel to aa opens. The a-boson will in turn decay to SM fermion pairs, with different branching ratios in different types of models and depending also on $\tan \beta$. Typically the decay to b quarks dominates above $10 \mathrm{GeV}$ followed by the decay to tau leptons, except in type-III models where it is reversed. The decay to muons is several order of magnitudes smaller but leading to a clean experimental signature.

With the run-I data, several decay modes were considered, covering different mass ranges, see for example [36]. With the run-II data, a new CMS analysis has been released studying $H \rightarrow a a \rightarrow$ $b b \tau \tau$ [37], exploiting the larger branching ratio to b quarks. The channel $H \rightarrow a a \rightarrow \mu \mu \tau \tau$ [38] has also been studied with the 2016 dataset. An example summary plot comparing the new results with the run-I results from [36] is shown in Figure 5, right.

ATLAS also released a novel analysis targeting the VBF production mode, and decay to gluon jets: $H \rightarrow a a \rightarrow \operatorname{gg} \gamma \gamma$ [39]. Similarly to the $4 \gamma$ decay mode, this channel targets models with fermionic decays suppressed, but with a much higher predicted branching ratio. The VBF production is selected by asking for 2 jets with an invariant mass $\mathrm{M}_{j j}>500 \mathrm{GeV}$. The $95 \% \mathrm{CL}$ upper limit on the branching ratio to gg $\gamma \gamma$ is shown in Figure 6, left.

\section{BSM Searches: neutral}

Additional neutral Higgs bosons are looked for with similar decay modes as the SM Higgs boson, in the high mass regions. A summary of the high-mass searches is given in Table 3. 



Figure 6: Left: $95 \% \mathrm{CL}$ upper limit on the branching ratio to $\mathrm{gg} \gamma \gamma$ as a function of the a boson mass, from [39]. Right: Observed significance in the search for heavy A bosons decaying to $\mathrm{VH}$, as a function of the mass of the A boson, from [42].

\begin{tabular}{|c|c|c|}
\hline Channel & ATLAS & CMS \\
\hline $\mathrm{H} \rightarrow \mathrm{ZZ}$ & arXiv:1712.06386 & arXiv:1804.01939 \\
\hline $\mathrm{H} \rightarrow \mathrm{WW}(\ell \ell v v)$ & Eur. Phys. J. C 78 (2018) 24 & JHEP 10 (2015) 144 (RunI) \\
\hline $\mathrm{H} \rightarrow \mathrm{WW}, \mathrm{WZ}(\mathrm{l} v \mathrm{qq})$ & JHEP 03 (2018) 042 & JHEP 10 (2015) 144 (RunI) \\
\hline $\mathrm{H} \rightarrow \tau \tau$ & JHEP 01 (2018) 055 & arXiv: 1803.06553 \\
\hline
\end{tabular}

Table 3: References for the ATLAS and CMS searches of high-mass neutral Higgs bosons.

Low mass Higgs boson decaying to $\gamma \gamma$ are searched for in the mass range $70<m_{H}<110$ $\mathrm{GeV}$ in [40]. A local (global) combined significance of 2.8 (1.3) $\sigma$ is seen for a mass of $95.4 \mathrm{GeV}$, not enough to conclude to an observation, but it is to be noted that an excess is seen separately in different production modes and datasets ( 8 and $13 \mathrm{TeV}$ datasets) in this same mass region. More data need to be analysed before a conclusion can be reached.

In addition to the standard decays to two SM bosons or two fermions, also cascade decays involving several Higgs bosons are possible depending on the scenarios. ATLAS has studied A bosons decaying to $\mathrm{ZH}$ in [41], targeting both gluon fusion and b-associated productions. Also decays of A bosons to a vector boson (W or Z) and SM Higgs bosons are studied by ATLAS in [42], with $\mathrm{W} \rightarrow \ell v, \mathrm{Z} \rightarrow \ell \ell, v v$, and $\mathrm{H} \rightarrow b \bar{b}$, and with resolved and merged topologies. Interpretations are given in heavy-vector-triplet and 2HDM models. A local excess is seen with a local (global) significance of 3.6 (2.4) $\sigma$, shown in Figure 6, right.

\section{Di-Higgs boson production}

Di-Higgs boson production in the SM is a non-resonant process with small cross sections $\mathrm{O} \simeq 30 \mathrm{fb}$ at $13 \mathrm{TeV}$. The cross section can be significantly enhanced in diverse BSM scenarios if resonant production becomes possible via new particles. Non-resonant enhancements are also considered, using description from Effective Field Theories (EFT). The run-I results are summarised in [43] and [44], for ATLAS and CMS respectively.

The branching ratios for di-Higgs boson production to different final states are shown in Figure 7, with the channels analysed already shown explicitely with red boxes. The run-II results 
references are summarised in the table in Figure 7. For non-resonant HH searches, the sensitivity reached is of the order of $20 \times \mathrm{SM}$. The EFT parametrisation is taken from [45].

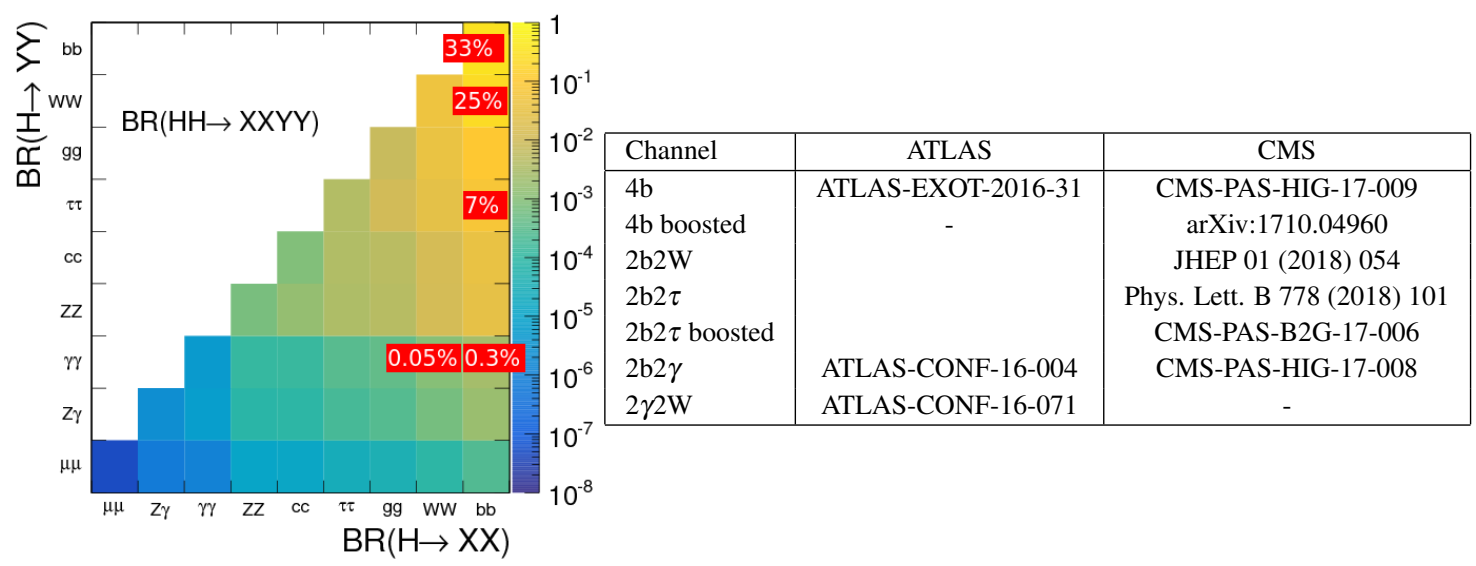

Figure 7: Branching ratios of di-Higgs boson production to different final states, and list of references for the run-II results.

\section{BSM Searches: charged}

Finding a charged Higgs boson would be an unequivoqual sign of physics beyond the SM. Many searches have been performed with unfortunately no signs of a charged Higgs boson yet in the data. References are summarised in Table 4 for ATLAS and CMS searches.

\begin{tabular}{|l|c|c|}
\hline Search & ATLAS & CMS \\
\hline $\mathrm{H}^{ \pm} \rightarrow c \bar{s} 8 \mathrm{TeV}$ & Eur. Phys. J. C, 736 (2013) 2465 & JHEP 12 (2015) 1 \\
$\mathrm{H}^{ \pm} \rightarrow c \bar{b} 8 \mathrm{TeV}$ & - & CMS-PAS-HIG-16-030 \\
$\mathrm{H}^{ \pm} \rightarrow t \bar{b} 8 \mathrm{TeV}$ & JHEP 03 (2016) 127 & JHEP 11 (2015) 018 \\
$\mathrm{H}^{ \pm} \rightarrow \tau \nu 13 \mathrm{TeV}$ & Phys. Lett. B 759 (2016) 555-574 & CMS-PAS-HIG-16-031 \\
$\mathrm{H}^{ \pm} \rightarrow t \bar{b} 13 \mathrm{TeV}$ & ATLAS-CONF-2016-089 & - \\
\hline $\mathrm{H}^{ \pm} \rightarrow W Z$ & Phys. Rev. Lett. 114, 231801 (2015) & PRL 119 (2017) 141802 \\
$\mathrm{H}^{ \pm \pm} \rightarrow \ell \ell \ell(\ell) 13 \mathrm{TeV}$ & Eur. Phys. J. C 78 (2018) 199 & CMS-PAS-HIG-16-036 \\
$\mathrm{H}^{ \pm \pm} \rightarrow W^{ \pm} W^{ \pm}$ & - & arXiv:1709.05822 \\
\hline
\end{tabular}

Table 4: References for all charged Higgs boson analyses in ATLAS and CMS.

\section{Conclusion}

With the analysis of the data taken in 2016 at a centre-of-mass energy of $13 \mathrm{TeV}$, the SM Higgs boson has now been observed in all production modes, including $\mathrm{ttH}$, and in the five main decay channels, with $5 \sigma$ discoveries in dibosons, $\gamma \gamma$ and $\tau \tau$, and $3 \sigma$ evidence for $b \bar{b}$. Coupling measurements are being completed in all production modes and decay channels. Concerning rare decays, the experiments are reaching $2.6 \times \mathrm{SM}$ in the $\mu \mu$ channel, and $4 \times \mathrm{SM}$ in rare decay to $\mathrm{Z} \gamma$. They are exploring decays to charm quarks and meson $+\gamma$ and also many exotic decay modes, e.g. to invisible particles or dark photons. 
The constraints from the visible decay channels and increasingly precise measurements achieved by both experiments are strong, but many different searches are developed to look for signs of new physics. In the objective of leaving no stone unturned, a good complementarity is seen between ATLAS and CMS. No significant-enough excess has been found yet leading to stronger and stronger constraints put on theoretical models.

\section{References}

[1] ATLAS Collaboration, "The ATLAS experiment at the CERN LHC", JINST 3:S08003,2008.

[2] CMS Collaboration, "The CMS experiment at the CERN LHC", JINST 3:S08004,2008.

[3] ATLAS Collaboration, https://twiki.cern.ch/twiki/bin/view/AtlasPublic/LuminosityPublicResultsRun2

[4] CMS Collaboration, https://twiki.cern.ch/twiki/bin/view/CMSPublic/LumiPublicResults

[5] ATLAS Collaboration, Phys. Lett. B 716 (2012) 1

[6] CMS Collaboration, PLB 716 (2012) 30

[7] ATLAS and CMS Collaborations, JHEP 08 (2016) 045

[8] CMS Collaboration, Phys. Lett. B 779 (2018) 283

[9] ATLAS Collaboration, JHEP 12 (2017) 024

[10] CMS Collaboration, Phys. Lett. B 780 (2018) 501

[11] ATLAS Collaboration, Phys. Rev. D 97 (2018) 072003

[12] CMS Collaboration, arXiv: 1804.02610

[13] ATLAS and CMS Collaborations, Phys. Rev. Lett. 114 (2015) 191803

[14] ATLAS Collaboration, ATLAS-CONF-2017-046, https://cds.cern.ch/record/2273853

[15] CMS Collaboration, JHEP 11 (2017) 047

[16] ATLAS Collaboration, Eur. Phys. J. C75 (2015) 476

[17] CMS Collaboration, Phys. Lett. B 775 (2017) 1

[18] Andrew Gilbert, LHC Seminar 10th April 2018, https://indico.cern.ch/event/682983

[19] CMS Collaboration, Phys. Rev. Lett. 120 (2018) 071802

[20] ATLAS Collaboration, ATLAS-CONF-2017-047, https://cds.cern.ch/record/2273854

[21] ATLAS Collaboration, ATLAS-CONF-2018-004, http://cdsweb.cern.ch/record/2308392

[22] CMS Collaboration, CMS-PAS-HIG-17-031, http://cds.cern.ch/record/2308127

[23] ATLAS Collaboration, ATLAS-CONF-2018-002, https://cds.cern.ch/record/2308390

[24] ATLAS Collaboration, arXiv:1802.04329

[25] ATLAS Collaboration, Phys. Rev. Lett. 119 (2017) 051802

[26] CMS Collaboration, CMS-PAS-HIG-17-019, http://cds.cern.ch/record/2292159

[27] ATLAS Collaboration, JHEP 10 (2017) 112 
[28] CMS Collaboration, CMS-PAS-HIG-17-007, http://cds.cern.ch/record/2308435

[29] ATLAS Collaboration, arXiv:1712.02758

[30] ATLAS Collaboration, Phys. Rev. Lett. 114 (2015) 121801

[31] CMS Collaboration, PLB 753 (2016) 341

[32] CMS Collaboration, Phys. Lett. B 749 (2015) 337

[33] CMS Collaboration, arXiv:1712.07173

[34] CMS Collaboration, CMS-PAS-HIG-17-023, http://cds.cern.ch/record/2308434

[35] ATLAS Collaboration, arXiv:1802.03388

[36] CMS Collaboration, JHEP 10 (2017) 076

[37] CMS Collaboration, CMS-PAS-HIG-17-024, http://cds.cern.ch/record/2306329

[38] CMS Collaboration, CMS-PAS-HIG-17-029, http://cds.cern.ch/record/2308654

[39] ATLAS Collaboration, arXiv:1803.11145

[40] CMS Collaboration, CMS-PAS-HIG-17-013, http://cds.cern.ch/record/2285326

[41] ATLAS Collaboration, arXiv:1804.01126

[42] ATLAS Collaboration, arXiv:1712.06518

[43] ATLAS Collaboration, Phys. Rev. D 92, 092004 (2015)

[44] CMS Collaboration, Phys. Rev. D 96, 072004 (2017)

[45] Alexandra Carvalho et al, arXiv:1608.06578 